\title{
DEVELOPMENT AND CLINICAL STUDY OF A PROJECTION IMAGES PROGRAM TO ENCOURAGE ARM MOVEMENTS FOR REHABILITATION
}

\author{
K. Yoshioka $\bowtie$ \\ Meisei University, Japan \\ $凶$ kiyomi.yoshioka@design.meisei-u.ac.jp
}

\begin{abstract}
In a program we developed called "Let's keep wiping to draw pictures!", projected graphic images change according to rehabilitation movements for upper limbs, and the levels of exercise amount and quality of movement achieved by patients are reflected in the outcome of the artwork as feedback. At a rehabilitation hospital, inpatients who used the program to perform rehabilitation exercises showed higher levels of satisfaction and expectation in the exercises, and performed simple and repetitive movements more willingly. The program can expect to maintain motivation towards rehabilitation.
\end{abstract}

Keywords: healthcare design, interaction design, design practice, rehabilitation

\section{Introduction}

Sustaining patient motivation to perform simple and repetitive exercises is a problem in rehabilitation aimed at the recovery of physical function. The outcome of recovery-phase rehabilitation work on stroke patients is improved by measures such as feeding back specific evaluation results, improving mood disorders, and providing social support. A study investigating the relationship between motivation and physical functions, psychological/mental aspects, social aspects, and the quality of life, has shown that motivation is related to daily life ability, independence, good communication skills, mood, and vitality (Kitaji et al., 2013).

In recent years, game-based rehabilitation devices have become available. Previous studies suggesting rehabilitation devices using game images have been shown to be effective in rehabilitation of hemiplegic stroke patients or fingers (Norma et al., 2016, Noveletto et al., 2018). Another previous studies reported rehabilitation effects using VR devices. In the VR rehabilitation, we can experience the real environment or play games (Borrego et al., 2017, Juras et al., 2018).

Previous studies of "Let's draw fireworks!" and "Let's draw jellyfish!" programs that make art works by touching a computer touch panel and squeezing a rubber air pump showed effects of improved mood and rehabilitation assistance (Yoshioka, 2015a, 2015b, 2016). These previous programs are expected to help maintain patients' motivation towards performing simple and repetitive rehabilitation exercises through the psychological effects of art, which occur when patients interact with art and the human senses are stimulated as a result of the interaction, and through the creative work of art making. Based on the previous studies, we developed the "Let's stand up and draw art in the sky!" program which included sitting-down and standing-up movements for rehabilitation. This program uses a Virtual Reality head 
mounted display. It connects rehabilitation exercises with the interactive image change, reflecting the level of rehabilitation exercise achieved in the resulting artwork as feedback (patented, Figure 1).

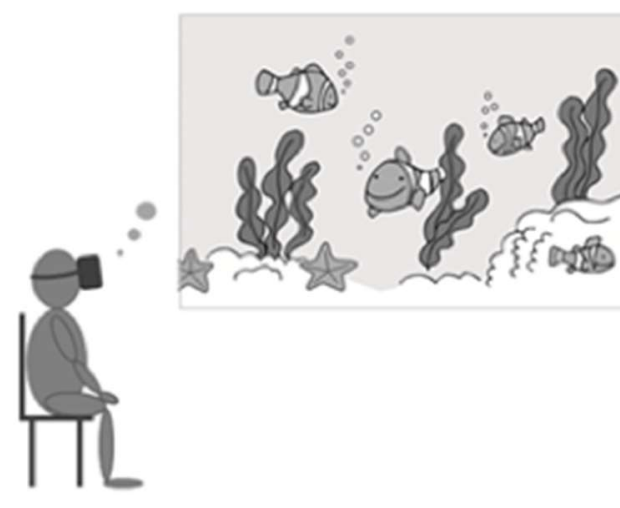

Display when sitting down

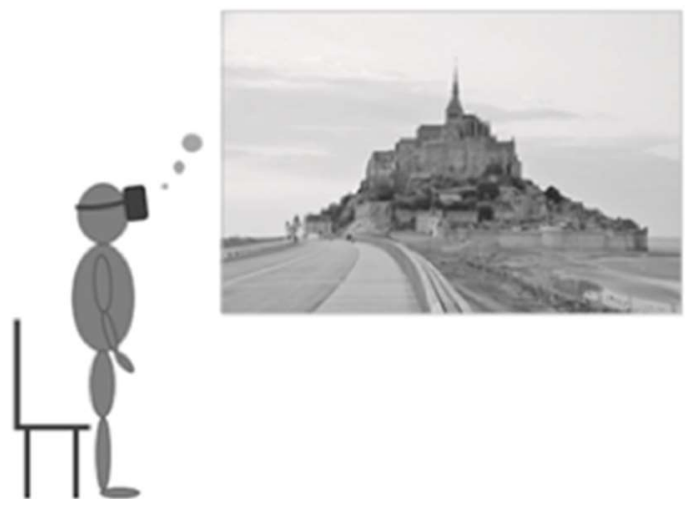

Display when standing up

Figure 1. "Let's stand up and draw art in the sky!" program

Psychological evaluations conducted on university students who performed squat exercises with and without the "Let's stand up and draw art in the sky!" program showed an increase in the students' levels of pleasure and vitality when they used the program. The participants gained comfortable and positive feelings by performing squat exercises with the program (Yoshioka, 2017, 2018a). In addition, our research results in a recovery-phase rehabilitation hospital indicate that the "Let's stand up and draw art in the sky!" program can help patients to maintain their motivation and to become actively involved in performing simple and repetitive rehabilitation exercises, thereby promoting recovery of physical function (Yoshioka, 2018b, 2019). Our new approach differs from other approaches using game machines (Norma et al., 2016, Noveletto et al., 2018) or experiencing virtual environments by VR (Borrego et al., 2017, Juras et al., 2018) in that the program maintains motivation through a combination of interactive image changes and creative activity, and promotes communication through the artwork produced as a result of rehabilitation exercises.

On the basis of our previous studies, the present study focused on a rehabilitation exercise known as sanding, and developed a program called "Let's keep wiping to draw pictures!" in which a projected image changes interactively according to the rehabilitative motion of flexing and extending the upper limbs. The aim of this study is to maintain patients' motivation towards performing simple and repetitive rehabilitation exercises, and thereby promote the recovery of physical function.

\section{Significance of sanding exercises for rehabilitation}

Sanding is a rehabilitation exercise involving flection and extension of the upper limbs, which is attained by sliding a hand-held board or towel in various directions on a horizontal or inclined tabletop (sanding board). Sanding exercises are used in various rehabilitation processes to improve functional disorders such as movement paralysis due to stroke, with the aim of recovering the function of paralyzed hands, strengthening upper limb muscles and improving joint movement.

\section{3. “Let's keep wiping to draw pictures!” program (patented)}

In the "Let's keep wiping to draw pictures!" program, an ultra-short focus projector (Epson EB-585W) and an input device (Web camera) are installed on the sanding board, and the image projected on the table changes gradually with the sanding motion until a graphic image is completed (patented, see Figure 2)

The program sets a target number of sanding movements (the maximum amount of exercise), and projects image pieces sequentially according to the number of movements (exercise amount) achieved, until the completion of a graphic image, when the target is met. At the beginning of the program, the start and end points of upper limb movement (a target distance of upper limb movement) are measured with a Web camera, and are established as a criterion for evaluation. 


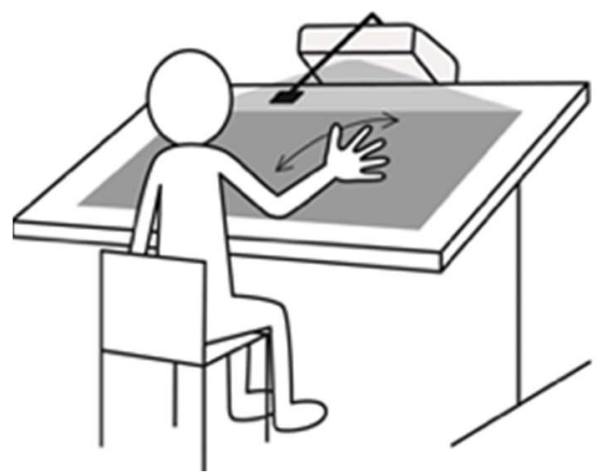

Figure 2. "Let's keep wiping to draw pictures!" program device (patented)

The level of achievement of the upper limb movement distance (the quality of movement) during the program is evaluated against the criterion, and fed back to the patient as reflected in the quality of the image projected on the table (e.g., resolution, number of colors, and drawing method) (Figure 3). The program also involves a creative element: after the end of the rehabilitation exercises, the graphic image created in relation to the levels of exercise amount and quality of movement achieved by the patient can be printed out on a postcard-sized piece of paper and taken home as a memento of the exercises performed. Reflecting the achievement levels of exercise amount and quality of movement in the resulting artwork, in terms of differences in the image, can improve the patient's motivation towards rehabilitation.
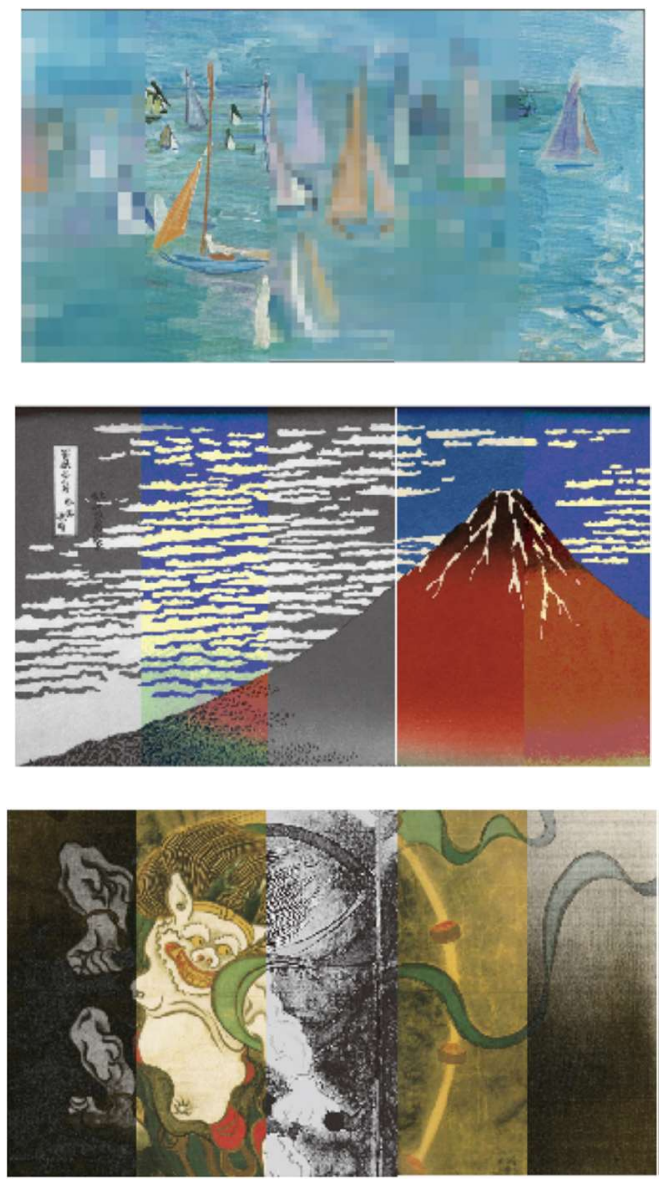

The achievement levels of quality of movement and exercise amount in the rehabilitation program are reflected in the outcome of the artwork as feedback (patented).

Figure 3. Projected image (example of completed artwork) 


\section{Formatting requirements: Clinical experiment involving rehabilitation using the "Let's keep wiping to draw pictures!" program}

\subsection{Methods}

A clinical experiment using the "Let's keep wiping to draw pictures!" program was conducted on 36 inpatients in a recovery-phase rehabilitation hospital, who needed to undergo rehabilitation for poststroke hemiplegia or fractures. Rehabilitation was conducted once a day for $10 \mathrm{~min}$, for 2 weeks (5 sessions a week excluding Saturdays and Sundays; 10 sessions in total). Among the patients who participated in the experiment, 15 performed exercises using the program and 21 performed the same exercises without using the program. In a recovery-phase rehabilitation hospital, intensive rehabilitation is conducted to recover patients' reduced capability; the patients are in a recovery phase (one to two months after onset), with their medical condition having begun to stabilize after surgery for cerebrovascular disorder or fracture as an acute phase treatment.

Strokes are often followed by depressive symptoms, and the influence of such symptoms makes it difficult to conduct psychological assessments using methods such as TDMS-ST and POMS. We therefore instead evaluated the patients' satisfaction and expectation levels in working on rehabilitation, on a seven-point scale. The satisfaction levels were: very satisfied, satisfied, rather satisfied, neutral, rather unsatisfied, unsatisfied, and very unsatisfied; and the expectation levels were very good expectation, good expectation, rather good expectation, neutral, rather poor expectation, poor expectation, and very poor expectation; each with corresponding scores of 7 to 1 , respectively.

Since functional recovery is more likely to occur as the number of rehabilitation movements increases, we also recorded the number of rehabilitation movements performed by each patient in 10 minutes. In addition, a graphic image reflecting the level of rehabilitation movement achieved by each patient was printed out on a postcard, and the patients took the printed image home as the outcome of the exercises. After the tenth rehabilitation session, we conducted a questionnaire survey to let the participants comment freely on the printed postcard.

\subsection{Results}

In assessing the patients' satisfaction and expectation in working on rehabilitation, verification was conducted using a three-factor two-level analysis of variance, and no significant differences were found in multiple comparisons between the presence and absence of the program, between the first and tenth sessions of rehabilitation, and between before and after rehabilitation exercises (Figures. 4 and 5). However, when rehabilitation exercises were performed without the program, the level of satisfaction decreased after exercising in both the first and tenth sessions; and when rehabilitation exercises were performed with the program, the level of satisfaction increased after exercising in both the first and tenth sessions. Furthermore, when rehabilitation exercises were performed without the program, the level of expectation after exercising became lower in the tenth session than in the first session; but when rehabilitation exercises were performed with the program, the level of expectation after exercising was higher in the tenth session than in the first session. Finally, the levels of satisfaction and expectation were higher when the program was used than when not used on all four before/after occasions: before and after the first rehabilitation session, and before and after the tenth session.

Figure 6 shows the progression in the average number of movements performed by the patients during each 10-min rehabilitation session. For the patients without the program, the average number of movements was the greatest in the fifth rehabilitation session, decreased in the sixth and seventh sessions, and then slightly fluctuated until the tenth session. For the patients with the program, the average number of movements increased from the first to fourth sessions, slightly fluctuated in the fifth to ninth sessions, and then became the greatest in the tenth session. 


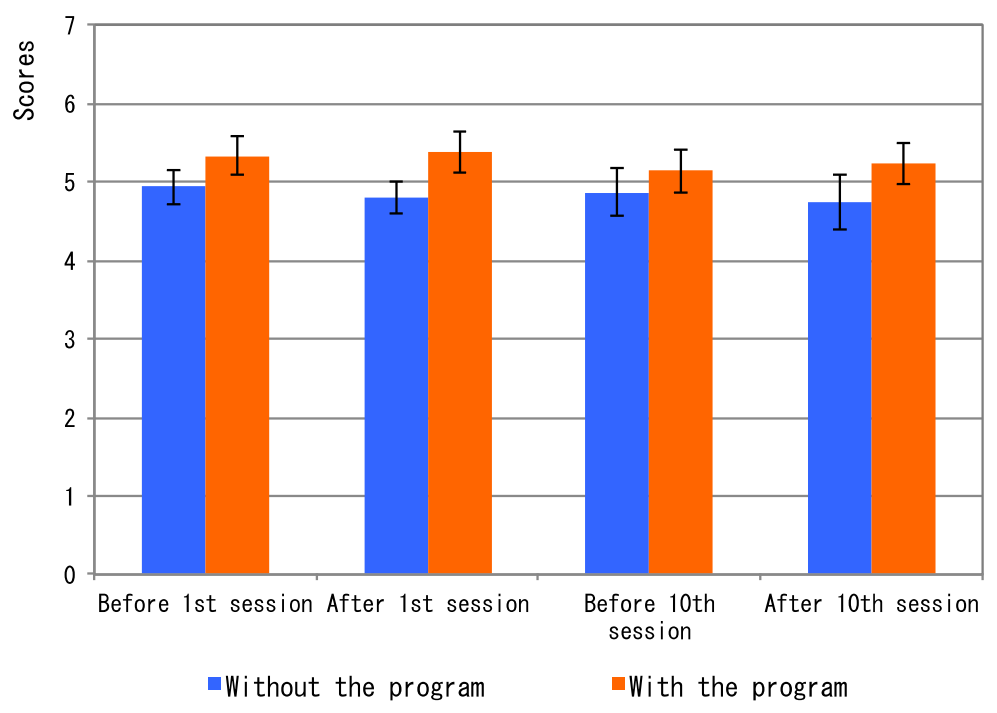

Figure 4. Comparison of average "Satisfaction" ratings in rehabilitation

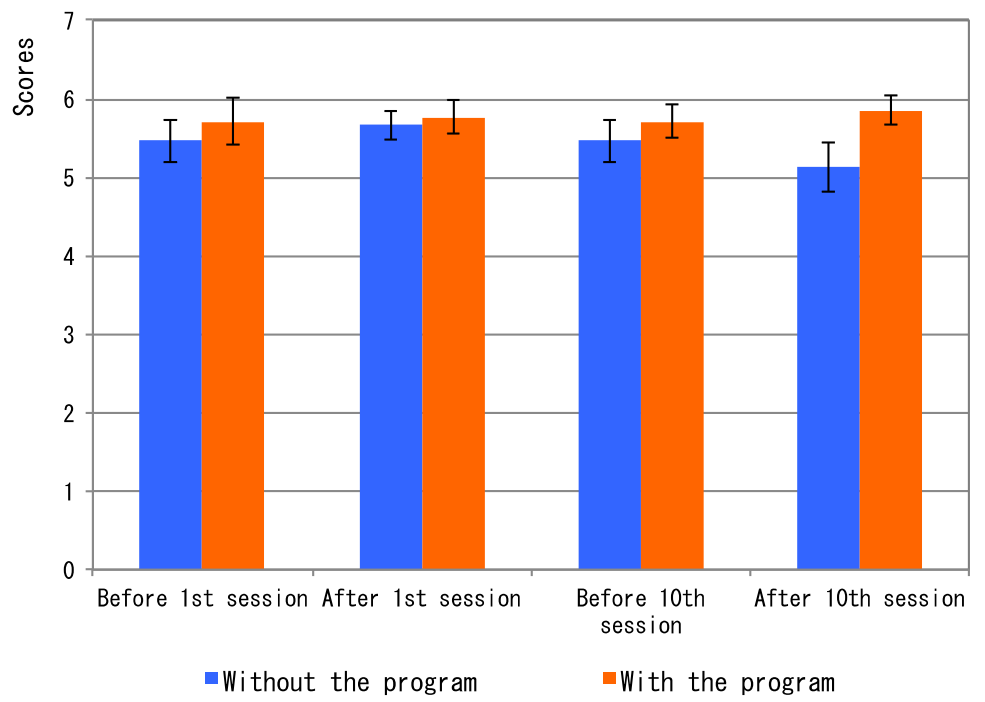

Figure 5. Comparison of average "Expectation" ratings in rehabilitation

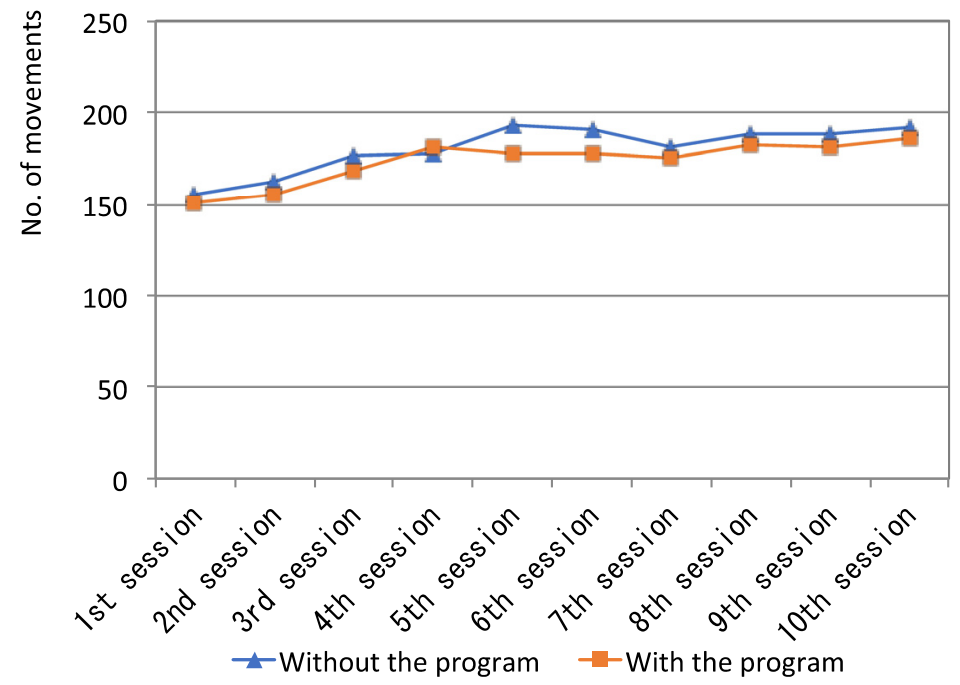

Figure 6. Change in the average number of arm flection and extension movements 
A graphic image reflecting the levels of exercise amount and quality of movement achieved by each patient was printed out on a postcard. In a questionnaire survey regarding the postcard, many patients responded positively; among the responses: "I find delight in seeing my postcard after performing rehabilitation exercises"; "I was satisfied to see the final image created"; "I have delight in seeing a postcard created as a result of my performing exercises"; "It was worth my effort/time"; "As the postcard is a reward for what I earnestly performed, I will buy a frame, put the postcard in it, and display it in my room"; "As it is fun to draw an image with great concentration, I will keep the postcard in a safe place"; "I find pleasure in seeing the outcome of my effort as an artwork"; "I will send this postcard to a friend"; "I will keep this postcard as a memory". On the other hand, only two patients felt they were unnecessary and did not print the images.

\subsection{Discussion}

At the recovery-phase rehabilitation hospital, patients who used the "Let's keep wiping to draw pictures!" program to perform sanding movements for rehabilitation showed higher levels of satisfaction and expectation, compared with patients who did not use the program. Among the patients who used the program, the levels of satisfaction and expectation were higher even before conducting the first and tenth rehabilitation sessions. This may reflect the patients' high expectations for conducting exercises in an unusual setting (in front of a sanding board on which a projector and a Web camera were installed, rather than before a plain sanding board). Among the patients in the program, the expected levels after the tenth rehabilitation session showed a tendency to be higher than after the first session. We therefore expect that the use of the program will encourage patients to actively engage in simple and repetitive rehabilitation exercises over a long period of time. Nevertheless, no significant differences were found in the statistical analysis, and further studies with extended implementation periods are necessary.

The average number of rehabilitation movements performed by the patients in the program slightly increased or decreased over a short time, but showed a general trend of gradual increase when exercises were continued over a longer period. These results suggest that continuous use of the program over a long period may lead to better recovery of physical function; an assumption which nonetheless should be verified in the future. On the other hand, the average number of movements by patients in the program was less than that of patients not in the program in all the sessions except the fourth. As the "Let's keep wiping to draw pictures!" program aims at practical rehabilitation, each exercise set is designed to involve 50 or 100 movements, and a new evaluation criterion must be established each time a given set is completed. The fact that the setup time was included in the experiment time may have affected the results obtained for those in the program. For a future clinical experiment, we must develop a program that can be conducted continuously throughout the time allocated for the experiment, or must deduct the setup time from the total experiment time in investigating the number of movements.

Regarding the printed postcard, many patients answered that the postcard led to enjoyment and satisfaction in working on rehabilitation. It would appear that the postcard, as a memento of the exercises performed, with its graphical image reflecting the achieved levels of exercise amount and quality of movement, contributes to maintaining the patients' motivation towards rehabilitation.

Overall, the results of clinical experimentation suggest that the "Let's keep wiping to draw pictures!" program can be used for sanding exercises to help patients' actively work on rehabilitation for the recovery of physical function.

\section{Conclusion}

We have developed the "Let's keep wiping to draw pictures!" program, which is used for sanding exercises involving flection and extension of the upper limbs. In this program, a graphic image projected on a table changes according to rehabilitation movements, and the levels of exercise amount and quality of movement achieved by patients are reflected in the outcome of the artwork as feedback. The program allows patients to work on rehabilitation while confirming the quality of movement and the exercise amount through the projected graphic image. 
At a recovery-phase rehabilitation hospital, inpatients who used the "Let's keep wiping to draw pictures!" program to perform rehabilitation exercises showed higher levels of satisfaction and expectation in the exercises, and performed simple and repetitive movements more willingly, compared with patients who did not use the program. The results suggest positive effects from the program, which reflects in the graphic image, as feedback, the achievement levels of quality of movement and exercise amount. Moreover, the level of expectation increased and the number of exercises gradually increased when rehabilitation exercises using the program were performed over a longer period. In the future, we plan to verify the effects of the program with extended implementation periods. A printed postcard with a graphic image may be provided to patients, after performing the exercises, only when they wish to have one; this option is generally expected to have the effect of creating motivation, such as enjoyment and satisfaction in working on rehabilitation.

The results of the clinical experiment suggest that the "Let's keep wiping to draw pictures!" program, which informs patients of their achievement levels of exercise amount and quality of movement as feedback, can be used in exercises involving flection and extension of the upper limbs, to maintain patients' motivation towards rehabilitation, and thereby promote the recovery of physical function.

This research was conducted with the approval of the Research Ethics Committee of Meisei University and the Research Ethics Committee of Fujita Health University Nanakuri Memorial Hospital.

Kiyomi Yoshioka (Meisei University) is the author of the patents related to the "Let's stand up and draw art in the sky!" program and the "Let's keep wiping to draw pictures!" program, as well as the inventor of the devices used in these programs.

\section{Acknowledgement}

This work was supported by JSPS KAKENHI Grant Number JP15H02881, JP19K12667. This research was performed with the cooperation of associate professor Toshiaki Uchiyama of University of Tsukuba in producing the device and of Fujita Health University Nanakuri Memorial Hospital in clinical study.

\section{References}

Kitaji, Y. et al. (2014), "Relationships with Quality of Life, Physical Function, and Psychological, Mental, and Social States of Stroke Patients in a Recovery Ward. Part III: Motivation for Rehabilitation”, The Society of Physical Therapy Science, Vol. 29 No. 6, pp. 1023-1026.

Norman, S.L. et al. (2016), "Movement Anticipation and EEG: Implications for BCI-Contingent Robot Therapy", IEEE Transactions on Neural Systems and Rehabilitation Engineering, Vol. 24 No. 8. https://doi.org/10.1109/TNSRE.2016.2528167

Noveletto, F. et al. (2018), "Biomedical Serious Game System for Balance Rehabilitation of Hemiparetic Stroke Patients", IEEE Transactions on Neural Systems and Rehabilitation Engineering, Vol. 26 No. 11. https://doi.org/10.1109/TNSRE.2018.2876670

Borrego, A. et al. (2017), "Comparison of Oculus Rift and HTC Vive: Feasibility for Virtual Reality-Based Exploration, Navigation, Exergaming, and Rehabilitation”, Games for Health Journal, Vol. 7 No. 3. https://doi.org/10.1089/g4h.2017.0114

Juras, G. et al. (2018), "Standards of Virtual Reality Application in Balance Training Programs in Clinical Practice: A Systematic Review", Games for Health Journal, Vol. 8 No. 2. https://doi.org/10.1089/ g4h.2018.0034

Yoshioka, K. (2015), "Mood Change Caused by 'Active Art' that Encourages Touching and Grasping Movements - Evaluation of when Operating the 'Let's Draw Fireworks!' Program”, International Journal of Affective Engineering, Vol. 14 No. 4, pp. 253-257.

Yoshioka, K. (2015), "Mood Change Caused by 'Active Art' that Encourages Touching and Grasping Movements - Psychological Effects and Practice in Pediatric Care Facilities of 'Let's Draw Fireworks!' Program", Bulletin of Japanese Society for the Science of Design, Vol. 62 No. 4, pp. 77-84. https://doi.org/10.5057/ijae.IJAE-D-14-27

Yoshioka, K. (2016), "Making of the Active Art Program that Encourages Touching and Grasping Movements", Proceedings of the 18th Conference of the Japan Society of Kansei Engineering, 2016, PJ41, Tokyo, Janan.

Yoshioka, K. (2017), "Psychological Effects of an Art Program with Feed Back Systems Reflecting Achievement Levels in Rehabilitation Exercises - Development of a VR Device Encouraging Squatting Movements", International Association of Societies of Design Research(IASDR2017), University of Cincinnati, USA. http://doi.org/10.7945/C23671 
Yoshioka, K. (2018), "Development and Psychological Effects of a VR Device Rehabilitation Program - Art Program with Feed Back Systems Reflecting Achievement Levels in Rehabilitation Exercises", Proceedings of the 7th International Conference on Kansei Engineering and Emotion Research(KEER2018), Kuching, Malaysia, pp. 538-546. https://doi.org/10.1007/978-981-10-8612-0_56

Yoshioka, K. (2018), "Development and Evaluation of a Virtual Reality Device Rehabilitation Program Psychological Effects of an Interactive Program with Feed Back Systems Reflecting Achievement Levels in Rehabilitation Movements of Sitting Down and Standing Up", Bulletin of Japanese Society for the Science of Design, Vol. 65 No. 1, pp. 35-40.

Yoshioka, K. (2019), "Development and Implementation of a VR Device Program to Encourage Standing and Sitting Movements for Rehabilitation", Proceedings of 7th International Conference for UD, The International Conference for Universal Design, March 4-6, 2019, International Association for Universal Design, Bangkok, Thailand. 